\title{
EFEITOS DO JOGO DE CÂMBIO NA CAPACIDADE FUNCIONAL E NA ATENÇÃO DE IDOSOS
}

\author{
Roseli Bess ${ }^{1}$ \\ Eliane Lucia Colussi \\ Ana Carolina Bertoletti De Marchi
}

resumo

Objetivo: Investigar os efeitos do jogo de câmbio na capacidade funcional e na atenção de idosos. Método: Trata-se de um estudo quase-experimental, com pré e pós-intervenção, em que participaram 21 idosos com idades entre 62 a 84 anos. Os jogos com duração de 60 minutos foram realizados duas vezes por semana durante 12 semanas. Para a avaliação da aptidão física foi utilizado o Protocolo Senior Fitness Test, que mede os parâmetros de força, flexibilidade, resistência aeróbica, velocidade, agilidade e equilíbrio. Para a avaliação da atenção sustentada e dividida foi utilizado o Teste de Trilhas Coloridas. Todos os participantes foram avaliados

1 Graduada em Educação Física. Mestre em Envelhecimento Humano. Professora da Prefeitura Municipal de Santa Rosa. E-mail: roselibess@gmail.com.

2 Graduada em História. Doutora em História. Professora Titular da Universidade de Passo Fundo (UPF), vinculada ao Programa de Pós-Graduação em Envelhecimento Humano (PPGEH). E-mail: colussi@upf.br.

3 Graduada em Bacharelado em Informática. Doutora em Informática na Educação. Professora Titular da Universidade de Passo Fundo (UPF), vinculada ao Programa de Pós-Graduação em EnveIhecimento Humano (PPGEH). E-mail: carolina@upf.br. 
no início da intervenção (pré) e após três meses (pós). Na análise dos dados foram utilizados os testes Postos com Sinais de Wilcoxon e Teste t de student para amostras pareadas. As associações entre variáveis numéricas foram avaliadas com o coeficiente de correlação de Pearson com valor de significância $p<0,05$. Resultados: Após três meses, o Índice de Aptidão Física Geral dos participantes apresentou melhoras significativas da pré para a pós-intervenção $\left(T_{\text {calc }}=4,5<\right.$ $\left.T_{\text {crtico }}=52\right)$, o que também ocorreu nos testes que avaliaram a força: levantar e sentar ( $p=0,004)$, flexão de antebraço $(p<0,001)$; mobilidade física: sentado, caminhar 2,44m e voltar a sentar ( $p=0,003)$; e resistência aeróbica: andar 6 minutos $(p<0,001)$. Na análise de atenção, os resultados mostraram que o jogo contribuiu para a melhora na atenção, indicando diferenças significativas nos dois testes da pré para a pós-intervenção (Forma 1 p<0,001 e Forma 2 p<0,001). Conclusão: O jogo de câmbio foi benéfico aos idosos pois apresentou uma melhora significativa da aptidão física e da atenção após a intervenção, o que o torna uma alternativa viável para a manutenção da capacidade funcional do idoso.

palavras-chave

Exercício Físico. Autonomia. Aptidão Física. Saúde.

Com o avançar da idade é natural ocorrer uma redução da capacidade funcional (CF), que pode limitar os idosos na realização de atividades cotidianas com autonomia e independência (MANSO; GALERA, 2015). Essa alteração do nível de CF está associada, ao declínio da aptidão física e ao comprometimento cognitivo, entre outros fatores (VILA et al., 2013; SILVA, COSTA, GUERRA, 2011; MACHADO et al., 2011). Uma das funções cognitivas afetadas pelo nível de CF é a atenção que, ao sofrer alterações com o aumento da idade, compromete a autonomia e independência do idoso (VILA et al., 2013).

A prática de exercício físico contribui de maneira significativa para a manutenção da aptidão física e, consequentemente, da capacidade funcional. De acordo com Camara et al. (2008), há uma tendência geral da avaliação da aptidão física para a identificação do potencial funcional do idoso. Nesse contexto, intervenções adaptadas de jogos esportivos podem proporcionar 
benefícios significativos na coordenação motora, mantendo e melhorando a aptidão física desses indivíduos (CAMPOS, NAKAMURA, KOKUBUN, 2016).

Os jogos adaptados surgem como possibilidade para favorecer a participação das pessoas que não conseguem fazê-los em modelo convencional, como é o caso do jogo de câmbio, voleibol adaptado aos idosos. Podem ser definidos como esporte modificado ou especialmente criado para atender às necessidades únicas dos indivíduos, dando aos jogadores condições de participação independente do condicionamento físico ou das habilidades desportivas, por meio da redução da intensidade do jogo (GORGATTI et al., 2008). Além disso, apresentam grande importância técnica-tática, fazendo com que o praticante elabore estratégias de ações de acordo com as técnicas adquiridas, o que favorece também as habilidades cognitivas (SILVA; GRECO, 2009).

Alguns estudos têm analisado os efeitos dos jogos adaptados sobre a capacidade funcional de idosos, sem considerar as habilidades cognitivas. Em Ueno et al. (2012), os participantes obtiveram melhora nos componentes: resistência de força, agilidade e equilíbrio dinâmico. No entanto, não apresentaram diferenças significativas na flexibilidade, assim como no estudo de Rebelatto et al. (2006). Berté et al. (2011) concluíram que o jogo adaptado teve efeito positivo nos valores médios da capacidade funcional, embora não tenha sido observada diferença significativa. Já Alfieri et al. (2009) apontaram que idosos praticantes de voleibol adaptado demonstraram mobilidade superior aos adultos sedentários. Nesses estudos, a prática de jogos adaptados proporcionou ganhos superiores na capacidade funcional dos praticantes, mesmo sem diferenças significativas.

Quanto à resistência aeróbica, à força e à flexibilidade dos membros inferiores e superiores, Vila et al. (2013) e Silva, Costa e Guerra (2011) comprovaram a importância de um programa de atividade física para a capacidade funcional de idosos. Em Vila et al. (2013), além dos aspectos funcionais, foi analisada a atenção em praticantes de exercício físico. Contudo, os autores não constataram melhora nesta função, ao contrário dos achados de Carreiro, Ferreira e Machado-Pinheiro (2009), que indicaram melhor desempenho em tarefas de orientação voluntária da atenção de homens universitários jogadores de voleibol. Tais achados evidenciam a necessidade de mais estudos que associem o aspecto da atenção nos jogos adaptados, para que seja possível analisar o favorecimento dessa habilidade cognitiva importante para a independência do idoso associado a sua capacidade funcional.

Diante desse cenário, esse trabalho teve como objetivo investigar os efeitos do jogo de câmbio na capacidade funcional e na atenção de idosos. O câmbio resultou da experiência de grupos de profissionais vinculados às Universidades 
e às prefeituras municipais, especialmente no sul do Brasil, justificando o baixo número de publicações internacionais. Consiste em uma adaptação do voleibol, praticado por adultos e idosos, na qual não é permitido saltar para evitar impactos e a bola é agarrada com as mãos e, após, arremessada para a quadra adversária. É uma modalidade que permite ao idoso se manter fisicamente ativo, pois facilita sua participação ao não exigir um esforço demasiado.

Durante o estudo buscou-se verificar: a resistência aeróbica, a mobilidade, a força muscular dos membros inferiores e superiores, a flexibilidade dos membros inferiores e superiores e a atenção dos participantes. A hipótese testada foi que a prática do jogo de câmbio pode melhorar a aptidão física e a atenção dos idosos praticantes e, consequentemente, sua capacidade funcional.

\section{Metodologia}

Trata-se de um estudo quase-experimental, com pré e pós-intervenção, aprovado pelo Comitê de Ética em Pesquisa por meio do parecer de número 569.688/14. Todos os participantes assinaram o Termo de Consentimento Livre e Esclarecido.

\subsection{Amostra}

Participaram do estudo 21 idosos, com idades entre 62 a 84 anos, residentes da região Noroeste do Rio Grande do Sul. Como critérios de inclusão foram considerados: ter idade superior a sessenta anos, perfil cognitivo normal, não possuir patologias neurológicas ou que comprometessem a realização das atividades e nunca ter praticado jogo de câmbio. Os participantes foram comunicados que mais de três faltas acarretaria no desligamento.

\subsection{Instrumentos}

Foram utilizados os seguintes instrumentos para coleta de dados:

a. Questionário sociodemográfico, com o objetivo de caracterizar o perfil do grupo e conhecer as condições de saúde e possíveis problemas em relação à atenção dos participantes. Esse questionário, composto por 13 questões fechadas, foi elaborado pela pesquisadora responsável, levando em consideração a literatura e os objetivos do estudo. 
b. Mini Exame do Estado Mental (MEEM) - Brucki et al. (2003), que tem por objetivo verificar a integridade das funções cognitivas. É composto por questões agrupadas em sete categorias: orientação temporal, orientação espacial, memória imediata, atenção e cálculo, evocação, linguagem e praxia construcional, com uma pontuação que pode variar de 0 a 30 pontos. A versão validada para a população brasileira e utilizada nessa pesquisa sugere pontos de corte relacionados aos anos de escolaridade dos indivíduos, ou seja, 20 pontos para analfabetos; 25 pontos para pessoas com escolaridade de 1 a 4 anos; 26,5 para 5 a 8 anos; 28 para 9 a 11 anos, e 29 anos para indivíduos com escolaridade superior a 11 anos.

c. Protocolo Sênior Fitness Test (SFT) - Rikli e Jones (2001), avalia os principais parâmetros físicos associados à mobilidade funcional em idosos (RIKLI; JONES, 1999), entre eles força, flexibilidade, resistência aeróbica, velocidade, agilidade e equilíbrio. Os testes são divididos em seis etapas: a) Levantar e sentar na cadeira: avalia a força e resistência dos membros inferiores. A pontuação é obtida pelo número de execuções corretas que o indivíduo realiza num intervalo de 30 segundos; b) Flexão de antebraço: avalia a força e resistência do membro superior. A pontuação é obtida pelo número de flexões corretas realizadas num intervalo de 30 segundos, usando um halter de $2 \mathrm{~kg}$ para as mulheres e de $4 \mathrm{~kg}$ para os homens; c) Sentado e alcançar: avalia a flexibilidade dos membros inferiores. $\mathrm{O}$ resultado corresponde à distância em centímetros que falta para tocar os dedos dos pés ou que consegue alcançar para além dos pés; d) Sentado e caminhar: avalia a mobilidade física (velocidade, agilidade e equilíbrio dinâmico). O resultado corresponde ao tempo que o indivíduo leva para caminhar 2,44m, voltar e sentar; e) Alcançar atrás das costas: avalia a flexibilidade dos membros superiores (ombro). O resultado corresponde a distância em centímetros que falta para as pontas dos dedos médios se tocarem ou da distância que os dedos conseguem se cruzar em sobreposição; f) Andar 6 minutos: avalia a resistência aeróbica. A pontuação é obtida da distância em metros percorrida em 6 minutos. A soma dos testes resulta no Índice de Aptidão Física Geral (IAFG). 
A classificação e a pontuação do IAFG são categorizadas por faixa etária, divididas em: 60-64 anos; 65-69 anos; 70-74 anos; 75-79 anos; 80-84 anos; 85-89 anos; 90-94 anos. Para realizar a pontuação é preciso somar os pontos de mulheres e homens separadamente. A classificação é dividida em cinco níveis: muito fraco, fraco, regular, bom e muito bom.

d. Teste de Trilhas Coloridas (TTC) - com adaptação brasileira de Rabelo et al. (2010), avalia a atenção sustentada e dividida. É dividido em Forma 1 e Forma 2. Ambas consistem em ligar os círculos na ordem correta, sem tirar o lápis do papel, o mais depressa possível. A diferença entre elas é que na Forma 2, além da troca de número, a cor também é alterada. A aplicação é individual e deve ser executada por um profissional da psicologia.

\subsection{Procedimentos}

Os participantes da pesquisa foram convidados por intermédio de pessoas já praticantes do jogo de câmbio. Todos realizaram a avaliação inicial com o MEEM com vistas a averiguar os critérios de inclusão, em especial a integridade das funções cognitivas.

Após, foram aplicados o questionário sociodemográfico, o Senior Fitness Test e o Teste de Trilhas Coloridas. A aplicação do Senior Fitness Test foi realizada de forma individual e assistida pela pesquisadora e com o auxílio de um estagiário de Educação Física. O Teste de Trilhas Coloridas foi administrado por uma psicóloga, que antes averiguou a acuidade visual para cores dos participantes, e o conhecimento da numeração, para identificar se o participante estava apto a realizar o teste. Após a intervenção, apenas o Senior Fitness Test e o Teste de Trilhas Coloridas foram utilizados na reavaliação dos participantes.

Todos os participantes realizaram os jogos de câmbio duas vezes por semana, durante um período de 12 semanas, totalizando 24 jogos, todos com 60 minutos de duração. Os primeiros quatro encontros foram destinados a orientações sobre posicionamento e regras do jogo. Os demais encontros foram organizados com o seguinte protocolo: 10 minutos de alongamento, 10 minutos de aquecimento, 10 minutos de exercícios com bola e 30 minutos de jogo. Em cada treino eram propostos novos desafios e níveis de dificuldade. Foram reforçados ao grupo os aspectos investigativos e a possibilidade de melhorar a capacidade funcional e a atenção, o que motivou a participação no projeto. 
O controle dos níveis de dificuldade era realizado a partir da observação da pesquisadora. Quando todos participantes conseguiam executar uma nova atividade solicitada, um novo desafio era proposto. Os desafios estavam relacionados ao aumento do nível de dificuldade, com exercícios mais forçados desde o aquecimento até os exercícios com bola, incentivando arremessos mais fortes e a movimentação mais rápida em quadra.

A cada jogo os participantes eram divididos em duas equipes, sendo que a escolha era feita de maneira mista em relação ao sexo e a idade para não haver vantagem ou desequilíbrio. As equipes eram dispostas em nove jogadores para cada lado. O sistema de rodízio era realizado continuamente para que todos os participantes tivessem o mesmo tempo em quadra. A rotação ocorria da seguinte forma: depois que o jogador da posição oito arremessava a bola, o jogador da posição nove saia da quadra para a entrada de um de fora na posição um. Os jogos eram praticados no Ginásio Municipal Pedro Deon na cidade de Santa Rosa.

\subsection{Análise de Dados}

Os dados do questionário sociodemográfico foram analisados por meio de estatística descritiva. Os resultados dos instrumentos Senior Fitness Test e Teste de Trilhas Coloridas foram analisados em planilha de Excel.

Na análise da aptidão em cada um dos exercícios, verificou-se que Alcançar as Costas não apresentou aderência à distribuição normal. Portanto, o teste aplicado para essa variável foi o de postos de sinais de Wilcoxon (TRIOLA, 2009).

$\mathrm{Na}$ análise do Senior Fitness Test, o teste comparativo foi não paramétrico porque houve dois participantes com resultados adversos devido a fatores estranhos ao programa (obesidade e perda de cônjuge). Esses acontecimentos poderiam ocasionar suposições para atingir a aderência à normalidade gaussiana. Também, o Senior Fitness Test faz ponderações dos resultados, de acordo com a idade e o sexo do participante. Diante disso, utilizou-se um método não paramétrico: teste dos postos de sinais de Wilcoxon. $\mathrm{O}$ valor calculado para o nível de significância $\alpha=0,05$ foi 4,5 , que comparado ao valor crítico tabelado, 52 , indica que existe evidência suficiente para rejeitar a hipótese nula (que os valores são iguais).

Para análise dos dados de atenção sustentada e dirigida, foi utilizado o método paramétrico Teste $t$ de student pareado, tendo em vista o número inferior a 30 participantes. Previamente foi utilizado o método do chi-quadrado para testar a aderência da distribuição com a distribuição normal. Para o teste 
de trilhas, Forma 1, o valor calculado do chi-quadrado foi 0,036 que foi comparado com o valor tabelado para a=0,05, com 4 graus s de liberdade, que é 9,49. Portanto, o método paramétrico pode ser utilizado. Do mesmo modo, para a Forma 2 os respectivos valores de chi-quadrado foram 1,99 e 5,99. As associações entre variáveis numéricas pré e pós-intervenção foram avaliadas utilizando-se o coeficiente de correlação de Pearson com valor de significância p<0,05.

\section{Resultados}

Os dados sociodemográficos e de caracterização dos participantes estão apresentados na Tabela 1 de acordo com a classificação por idade definida pelo Índice de Aptidão Física Geral (IAFG). Dentre os participantes, 76,1\% iniciaram o ensino fundamental sem conclúi-lo, estudaram no máximo cinco anos; apenas $4,7 \%$ concluíram o ensino médio; e 19,0\% concluíram o ensino superior.

Dos 21 participantes da pesquisa, $71,4 \%$ eram do sexo feminino e $28,5 \%$ do sexo masculino, com idade média de $68,61 \pm 5,74$ anos. Todos os homens eram casados. Das mulheres, seis eram viúvas, seis casadas e três divorciadas.

Em relação às doenças pré-existentes, $76,1 \%$ relataram ter pelo menos um problema de saúde. Dentre as citados estão: hipertensão arterial (52,3\%), doenças coronarianas $(19,0 \%)$, artrose $(9,5 \%)$, diabetes $(4,7 \%)$, problema de audição $(4,7 \%)$ e catarata $(4,7 \%)$. Todos que relataram problemas de saúde faziam uso de um ou mais tipos de medicamentos.

Apenas uma pessoa declarou não ser aposentada, mas também não exercia atividade laboral fora de casa e a renda advinha do trabalho do marido. Os outros eram aposentados e pensionistas, com rendimentos entre 2 a 3 salários.

O grupo não era sedentário, 80,9\% já praticava alguma atividade física, como caminhadas, ginástica e bicicleta, pelo menos duas a três vezes por semana. Quanto à atenção, 66,6\% declararam possuir dificuldades de concentração. 
Tabela 1 - Características sociodemográficas da amostra.

\begin{tabular}{|c|c|c|c|c|c|c|}
\hline Faixa de idade & $60-64$ & $65-69$ & $70-74$ & $75-79$ & $80-84$ & Total \\
\hline Participantes & 4 & 11 & 3 & 1 & 2 & 21 \\
\hline Sexo Masculino & 2 & 1 & 1 & 1 & 1 & 6 \\
\hline Sexo Feminino & 3 & 9 & 2 & - & 1 & 15 \\
\hline $\begin{array}{l}\text { Escolaridade } \\
\text { fundamental }\end{array}$ & 3 & 9 & 1 & 1 & 2 & 16 \\
\hline Escolaridade média & - & 1 & - & - & - & 1 \\
\hline $\begin{array}{l}\text { Escolaridade } \\
\text { superior }\end{array}$ & 1 & 1 & 2 & - & - & 4 \\
\hline $\begin{array}{l}\text { Doenças } \\
\text { pré-existentes }\end{array}$ & 2 & 9 & 2 & 1 & 2 & 16 \\
\hline $\begin{array}{l}\text { Uso de } \\
\text { medicamentos }\end{array}$ & 2 & 9 & 2 & 1 & 2 & 16 \\
\hline $\begin{array}{l}\text { Pratica exercícios } \\
\text { físicos }\end{array}$ & 4 & 8 & 3 & 1 & 1 & 17 \\
\hline $\begin{array}{l}\text { Dificuldade de } \\
\text { atenção }\end{array}$ & 2 & 9 & 1 & 1 & 1 & 14 \\
\hline
\end{tabular}

Nota: Idades definidas pelo Índice de Aptidão Física Geral (IAFG). Valores expressam frequência absoluta.

Fonte: Tabela elaborada pelas autoras.

O Índice de Aptidão Física Geral (IAFG), caracterizado pela soma de todos os testes do Senior Fitness Test, obteve valor do parâmetro T calculado de 4,5 e o valor crítico tabelado 52. Nesse caso, a hipótese nula foi rejeitada, confirmando que existe diferença de desempenho antes e depois da intervenção. Dos 21 participantes, 19 melhoraram o seu IAFG, conforme Tabela 2. 


\begin{tabular}{|c|c|}
\hline \multicolumn{2}{|c|}{ IAFG } \\
\hline Antes & Depois \\
\hline 40,0 & 62,5 \\
\hline 60,0 & 72,5 \\
\hline 32,5 & 55,0 \\
\hline 52,5 & 57,5 \\
\hline 47,5 & 52,5 \\
\hline 60,0 & 47,5 \\
\hline 45,0 & 72,5 \\
\hline 50,0 & 62,5 \\
\hline 35,0 & 60,0 \\
\hline 27,5 & 50,0 \\
\hline 30,0 & 30,0 \\
\hline 37,5 & 50,0 \\
\hline 47,5 & 72,5 \\
\hline 52,5 & 55,0 \\
\hline 47,5 & 50,0 \\
\hline 45,0 & 37,5 \\
\hline 27,5 & 30,0 \\
\hline 25,0 & 47,5 \\
\hline 62,5 & 85,0 \\
\hline 47,5 & 70,0 \\
\hline 45,0 & 57,5 \\
\hline
\end{tabular}

Fonte: Tabela elaborada pelas autoras.

A Tabela 3 apresenta os resultados dos parâmetros de força, flexibilidade, resistência aeróbica, velocidade, agilidade e equilíbrio do Senior Fitness Test. Os resultados indicaram diferenças significativas entre a pré e a pós- intervenção para os parâmetros de força: levantar e sentar $(\mathrm{p}=0,004)$ e flexão de antebraço $(\mathrm{p}<0,001)$; de mobilidade física: sentado e caminhar $(\mathrm{p}=0,003)$; e de resistência aeróbica: andar seis minutos $(\mathrm{p}<0,001)$. 


\begin{tabular}{cccccc} 
CF & \multicolumn{2}{c}{ Pré-intervenção } & \multicolumn{2}{c}{ Pós-intervenção } & p \\
& M & DP & M & DP & 0,004 \\
\hline LS & 12,2 & 1,5 & 14,1 & 3,1 & $<0,001$ \\
FA & 15,5 & 3,4 & 19,8 & 4,7 & 0,310 \\
AS & 4,9 & 8,1 & 6,1 & 7,4 & 0,003 \\
SC & 6,6 & 1,0 & 5,7 & 1,1 & $T_{\text {calc }}=34>T_{c o}=21$ \\
AC & 6,6 & 12,6 & 5,7 & 12,1 & $<0,001$ \\
A6 & 497,0 & 43,0 & 534,3 & 49,1 &
\end{tabular}

Nota 1: LS: Levantar e sentar; FA: Flexão de antebraço; AS: Sentado e alcançar; SC: Sentado e caminhar; AC: Alcançar atrás das costas; A6: Andar 6 minutos; M: Média; DP: Desvio padrão.

Nota 2: Teste t de Student valor significativo para um $\mathrm{p} \leq 0,05$ para LS, FA, AS, SC e A6. Teste dos postos de sinais para AC.

Nota 3: Unidades: LS- repetições em 30 s; FA- repetições em 30s; SA- cm; SC- segundos; AC$\mathrm{cm} ; \mathrm{A6}-\mathrm{m}$.

Fonte: Tabela elaborada pelas autoras

A Tabela 4 apresenta os resultados da análise do Teste de Trilhas Coloridas. Os resultados indicaram diferenças significativas $(\mathrm{p}<0,001)$ para os dois testes de análise de atenção dos participantes, Forma 1 e Forma 2.

Tabela 4 - Análise da atenção sustentada e dividida.

\begin{tabular}{cccccc}
\hline \multirow{2}{*}{ ASD } & \multicolumn{2}{c}{ Testes } & & \\
& Pré-intervenção & & Pós-intervenção & p \\
& M & DP & M & DP & \\
\hline Forma 1 & 97,0 & 58,1 & 68,0 & 48,6 & $<0,001$ \\
Forma 2 & 165,3 & 53,5 & 114,5 & 47,4 & $<0,001$ \\
\hline
\end{tabular}

Nota: M: Média; DP: Desvio padrão; Teste t de Student; valor significativo para um $p \leq 0,05$. Unidade: segundos

Fonte: Tabela elaborada pelas autoras.

\section{Discussão}

Os resultados encontrados no Índice de Aptidão Física Geral revelaram melhora significativa da pré para a pós-intervenção, corroborando com os achados de Campos, Nakamura e Kokubun (2016), que também analisaram essa variável em jogos de voleibol adaptado. No entanto, um dos elementos 
importantes da aptidão física é a flexibilidade, a qual não se identificou melhora significativa nesse estudo, da mesma forma que as pesquisas de Ueno et al. (2012) e Rebelatto et al. (2006). O primeiro analisou os efeitos de três modalidades de atividade física para verificar a relação com a capacidade funcional. No segundo, os autores apontaram que a elasticidade dos tendões, ligamentos e cápsulas articulares diminuem com a idade devido à deficiência de colágeno, determinando que durante a vida ativa, adultos perdem algo como 8 a $10 \mathrm{~cm}$ de flexibilidade na região lombar e no quadril, quando medido por meio do teste de sentar e alcançar, o mesmo utilizado nesse estudo. Com o processo de envelhecimento é inevitável a redução progressiva da flexibilidade. Nos estudos citados, assim como nesse, não foi planejado um programa de treinamento que incorporasse sessões com maior tempo de duração e repetições para o alongamento. Além disso, acredita-se que seja necessário um tempo maior de intervenção para que os efeitos possam ser comprovados também na flexibilidade.

No teste de levantar e sentar da cadeira, que avalia a força e a resistência dos membros inferiores, constatou-se melhoras significativas em relação a pré e a pós-intervenção. Tais achados confirmam os de Silva, Costa e Guerra (2011), no qual os participantes do programa de ginástica tiveram a média melhor em relação aos não praticantes nestes aspectos. $\mathrm{O}$ câmbio requer força de membros inferiores em pelo menos dois movimentos constantes durante o jogo, o arremesso da bola e se abaixar para pegá-la.

No teste flexão de antebraço apenas duas pessoas não aumentaram o número de repetições da pré para a pós-intervenção. Constatou-se que no quesito força dos membros superiores, o jogo de câmbio teve mais influência comparada ao dos membros inferiores. Durante o jogo os braços são muito utilizados, chegando o jogador a executar aproximadamente 34 arremessos de bola durante uma partida de uma hora, exigindo constantemente a força dos membros superiores. Tal fato foi comprovado também por Pereira et al. (2015), que avaliou a força dos membros superiores em 20 jogadoras jovens de voleibol e concluiu que a modalidade favoreceu tal resultado.

No teste sentado, caminhar 2,44m e voltar a sentar, que avalia a mobilidade física, a diferença também foi significativa. Apenas quatro pessoas não conseguiram diminuir o tempo do exercício, da primeira para a segunda execução, mas com diferenças quase imperceptíveis. Os resultados foram similares ao estudo de Alfieri et al. (2009) que comparou e avaliou a mobilidade funcional de 70 idosos praticantes de voleibol adaptado para idosos, e constatou que os mesmos realizaram o teste em um tempo menor do que o grupo de idosos sedentários e adultos jovens. De acordo com os autores, o voleibol adaptado 
proporciona estímulos músculos-esqueléticos e proprioceptivos, que contribuem para a melhora da mobilidade física.

No teste andar 6 minutos, em um percurso de $45,7 \mathrm{~m}$, que avalia a resistência aeróbica, importante para as pessoas conseguirem realizar atividade da vida diária, também observou-se uma diferença significativa em relação a pré e a pós-intervenção, assim como no estudo de Vila et al. (2013). Os autores também utilizaram o Senior Fitness Test nas 25 idosas participantes, que foram divididas em praticantes de exercício físico e sedentárias. A melhora da resistência aeróbica no câmbio deve-se ao fato do jogo exigir movimentação constante em quadra para a execução dos movimentos básicos.

Na análise do teste de trilhas coloridas, que verificou o nível de atenção dos participantes, as diferenças foram mais significativas em relação ao teste de aptidão física. Na Forma 1 apenas dois participantes não obtiveram diferenças da pré para a pós-intervenção, um deles permaneceu com o mesmo tempo de execução e o outro aumentou o tempo de execução do teste de $75 \mathrm{~s}$ para 90s. Na Forma 2, os resultados foram ainda mais significativos, tendo todos os participantes diminuído consideravelmente o tempo de realização do teste. Esse teste é mais complexo do que o Forma 1 e recruta outras funções, como atenção alternada, flexibilidade e comportamento inibitório. Para tanto, necessita de mais tempo para sua realização e, mesmo assim, os participantes apresentaram melhores resultados e cometeram menos erros em relação à primeira aplicação. Os achados quanto à atenção corroboram com o estudo de Carreiro, Ferreira e Machado-Pinheiro (2009), em que os atletas apresentaram melhor desempenho em tarefas de orientação voluntária da atenção, comparado aos não atletas, o que se deve aos estímulos recebidos no jogo. No entanto, diferem dos resultados de Vila et al. (2013), no qual os participantes não obtiveram melhora na atenção, justificado pelo fato das atividades físicas não exigirem conhecimento e evocação constante de regras.

No jogo de câmbio a atenção é muito exigida durante os treinos, já que os participantes precisam elaborar estratégias de ação para vencer, que envolvem estar atentos e concentrados no movimento da bola para que não caia em sua quadra e, ainda, fazer o ponto no lado adversário. Além disso, também demanda raciocínio e conhecimento por parte dos praticantes quanto às regras. De acordo com Miranda e Farias (2008), exercícios praticados por idosos devem envolver decisão, deslocamento, resolução de problemas, memorização de espaço e de tempo, atividades de percepção do corpo, estimulando e desafiando as capacidades intelectuais, além das capacidades físicas. É preciso que seja desenvolvido um trabalho sem que o participante apenas imite ou repita mecanicamente um movimento. 
O Índice de Aptidão Física Geral e a atenção melhoraram significativamente com a prática do jogo de câmbio nos idosos avaliados. As diferenças foram significativas nos aspectos relacionados à força, à mobilidade física, à resistência aeróbica e à atenção. Esse desfecho confirma a hipótese de que o jogo de câmbio pode ser uma alternativa para a manutenção da capacidade funcional, uma vez que auxilia na aptidão física e na atenção das pessoas idosas. A prática de uma atividade física como o câmbio pode auxiliar na promoção da autonomia e da independência de idosos, diminuir a dependência funcional e promover um envelhecimento ativo por meio da realização das atividades cotidianas.

Por fim, são necessários mais estudos sobre os jogos adaptados para idosos. Recomenda-se, com base nas limitações desse estudo, a realização de um ensaio clínico randomizado para que seja possível comparar os resultados com um grupo controle.

\section{EFFECTS OF EXCHANGE VOLLEYBALL GAME IN ELDERLY FUNCTIONAL CAPACITY AND ATTENTION}

abstract

Objective: To investigate the effects of adapted volleyball on the functional capacity and attention of the elderly. Method: It is a quasi-experimental study, with pre and post-intervention, involving 21 elderly people aged 62 to 84 years. The games were held twice a week for 12 weeks, lasting 60 minutes. The Senior Fitness Test Protocol was used for Physical fitness assessment. It measures the parameters of strength, flexibility, aerobic endurance, speed, agility and balance. For the evaluation of the sustained and divided attention, the Test of Colored Trails was used. All participants were evaluated at the beginning of the intervention (pre) and after three months (post). The Wilcoxon Signal Test and the Student T test for paired samples were used for data analysis. The associations between numerical variables were assessed using Pearson's correlation coefficient with significance value $<0.05$. Results: After three months, the participants' IAFG showed significant improvements from pre-post-intervention $\left(T_{\text {calc }}=4.5<T_{\text {critic }}=52\right)$, which also occurred in the tests that evaluated strength: lift and sit ( $p=0.004)$, forearm flexion ( $p<0.001)$; physical 
mobility: sitting, walking 2.44m and sitting again ( $p=0.003$ ); and aerobic

endurance: walking 6 minutes ( $p<0.001)$. In the analysis of attention, the results showed that the game contributed to the improvement in attention, indicating significant differences in the two tests from pre to post-intervention (Form $1 \mathrm{p}<0.001$ and Form $2 \mathrm{p}<0.001$ ). Conclusion: The adapted volleyball was beneficial to the elderly due to the significant improvement in physical fitness and attention after the intervention, which makes it a viable alternative for the maintenance of the functional capacity of the elderly.

keywords

Physical exercise. Autonomy. Physical fitness. Health.

referências

ALFIERI, Fábio Marcon et al. Mobilidade funcional de idosos ativos e sedentários versus adultos sedentários. Brazilian Journal of Biomotricity, Itaperuna, v. 3, n. 1, p. 89-94, mar. 2009.

BERTÉ, Jéssica Karine et al. Efeitos de seis meses de jogos adaptados na composição corporal, capacidade funcional e no sistema imunológico de idosos. Revista Brasileira de Prescrição e Fisiologia do Exercício, São Paulo, v. 5, n. 30, p. 503-509, nov./dez. 2011.

BRUCKI, Sonia et al. Sugestões para o uso do mini-exame do estado mental no Brasil. Arquivos de Neuro-Psiquiatria, São Paulo, v. 63, n. 3B, p. 777-81, set. 2003.

CAMARA, Fabioano Marques et al. Capacidade funcional do idoso: formas de avaliação e tendências. Acta Fisiátrica, São Paulo, v. 15, n. 4, p. 249-256, 2008. Disponível em: <http://www.revistas.usp.br/actafisiatrica/article/view/103005>. Acesso em: 13 abr. 2018.

CAMPOS, Leonardo; NAKAMURA, Priscila; KOKUBUN, Eduardo. Comparison of physical fitness in elderly submitted to different physical exercise interventions. Revista Brasileira de Atividade Física e Saúde, Florianópolis, v. 21, p. 78-84, 2016.

CARREIRO, Luiz Renato Rodrigues; FERREIRA, lara Ribeiro; MACHADO-PINHEIRO, Walter. Comparação de desempenho de jogadores de voleibol e não esportistas em tarefas de orientação automática e voluntária da atenção visual: um estudo exploratório. Psicologia: teoria e prática, São Paulo, v. 11, n. 2, p. 38-49, 2009.

GORGATTI, Márcia Greguol et al. Tendência competitiva no esporte adaptado. Arquivos Sanny de Pesquisa e Saúde, Londrina, v. 18, n. 1, p. 18-25, 2008.

MACHADO, Juliana Costa et al. Declínio cognitivo de idosos e sua associação com fatores epidemiológicos em Viçosa, Minas Gerais. Revista Brasileira de Geriatria e Gerontologia, Rio de Janeiro, v. 14, n. 1, p. 109-121, 2011.

MANSO, Maria Elisa Gonzalez; GALERA, Priscila Bovolini. Perfil de um grupo de idosos participantes de um programa de prevenção de doenças crônicas. Estudos Interdisciplinares sobre o Envelhecimento, Porto Alegre, v. 20, n. 1, p. 57-71, abr. 2015.

MIRANDA, Letícia Miranda de; FARIAS, Sidney Ferreira. A influência do exercício físico na atividade cerebral do idoso. Lecturas: educación física y deportes, Buenos Aires, v. 13, n. 125 , oct. 2008. 
PEREIRA, Ana et al. Training strategy of explosive strength in young female volleyball players. Medicina, Kaunas, v. 51, n. 2, p. 126-131, Apr. 2015.

RABELO, Ivan Sant'Ana et al. Teste de trilhas coloridas. São Paulo: Casa do Psicólogo, 2010.

REBELATTO, José Rubens et al. Influência de um programa de atividade física de longa duração sobre a força muscular manual e a flexibilidade corporal de mulheres idosas. Brazilian Journal of Physical Therapy, São Carlos, v. 10, n. 1, p. 127-132, 2006.

RIKLLI, Roberta; JONES, Jessie. The reliability and validity of a 6- minute walk test as a measure of physical endurance in older adults. Journal of Aging and Physical Activity, v. 6, n. 4, p. 363-375, 1999.

Senior fitness test manual. Illinois: Human Kinetics, 2001.

SILVA, Marcelo Vilhena; GRECO, Pablo Juan. A influência dos métodos de ensino -aprendizagem-treinamento no desenvolvimento da inteligência e criatividade tática em atletas de futsal. Revista Brasileira de Educação Física e Esporte, São Paulo, v. 23, n. 3, p. 297-307, jul./set. 2009.

SILVA, Tânia Cristina Lima da; COSTA, Eduardo Caldas; GUERRA, Ricardo Oliveira. Resistência aeróbica e força dos membros inferiores de idosos praticantes e não-praticantes de ginástica recreativa em um centro de convivência. Revista Brasileira Geriatria e Gerontologia, Rio de Janeiro, v. 14, n. 3, p. 535-542, 2011.

TRIOLA, Mario. Introdução à Estatística. Rio de Janeiro: LTC, 2009.

UENO, Deisy Terumi et al. Efeitos de três modalidades de atividade física na capacidade funcional de idosos. Revista Brasileira de Educação Física e Esporte, São Paulo, v. 26, p. 273-281, jun. 2012

VILA, Camila Prats et al. Aptidão física funcional e nível de atenção em idosas praticantes de exercício físico. Revista Brasileira de Geriatria e Gerontologia, Rio de Janeiro, v. 16, n. 2, p. 355-364, 2013. 